\title{
INVERSE SCATTERING FOR THE KLEIN-GORDON EQUATION
}

\author{
BY G. PERLA MENZALA
}

Communicated by Richard K. Miller, January 26, 1977

In this note we would like to announce recent results concerning the socalled Inverse Scattering problem for the Klein-Gordon equation in three dimensions. Complete proofs of this work will appear in [1].

We consider the Klein-Gordon equation with a linear perturbation, that is

$$
u_{t t}-\Delta u+m^{2} u+q(x) u=0
$$

in $\Omega=\mathbf{R}^{3},-\infty<t<+\infty$. Here the subscripts denote partial derivatives, $m>$ 0 and $\Delta$ is the Laplacian operator. The potential $q(x)$ is assumed to be a real valued function in $\mathbf{R}^{\mathbf{3}}$, nonnegative and satisfying certain reasonable conditions at infinity which we will specify later. The initial Cauchy data for (1) at $t=0$ will be assumed to be $C^{\infty}$ with compact support. In the space of such solutions of (1) we define the (total) energy of $u$ as

$$
\|u\|_{E}^{2}=\frac{1}{2} \int_{\mathbf{R}^{3}}\left[|\operatorname{grad} u|^{2}+u_{t}^{2}+m^{2} u^{2}+q(x) u^{2}\right] d x
$$

where $|\operatorname{grad} u|^{2}=\Sigma_{j=1}^{3} u_{x_{j}}^{2}$ It is easy to show that $\|u\|_{E}$ is constant i.e. we are dealing with a conservative equation. If we assume (for example) that $q(x) \in$ $L^{1} \cap L^{\infty}\left(\mathbf{R}^{3}\right)$ then it is well known (see for example [3] and [4]) that given a solution $u$ of (1) there then exists a unique pair $u_{ \pm}$of solutions of (1) with $q \equiv$ 0 such that

$$
\left\|u-u_{ \pm}\right\|_{E} \rightarrow 0 \text { as } t \rightarrow \pm \infty .
$$

The operator which relates $u_{-} \rightarrow u_{+}$is called the scattering operator and is denoted by $S$. One want to know what can be said about $q(x)$ if we know the operator $S$ ? This is a problem of physical relevance (see [5], [6]). If $q(x)$ is spherically symmetric, then there has been considerable research on this problem in the past twenty five years, mainly through the Gelfand-Levitan-Marchenko approach. In dimensions higher than one, very little is known. Here, we announce a "local" uniqueness result concerning the 3-dimensional inverse problem for (1).

THEOREM. Let $q_{1}(x)$ and $q_{2}(x)$ be a nonnegative continuous functions which belong to $L^{1} \cap L^{\infty}\left(\mathbf{R}^{3}\right)$. Let $S\left(q_{1}\right)$ and $S\left(q_{2}\right)$ denote the scattering operators associated with $u_{t t}-\Delta u+m^{2} u+q_{1} u=0$ and $v_{t t}-\Delta v+m^{2} v+q_{2} v=0$ 
respectively. If $S\left(q_{1}\right)=S\left(q_{2}\right)$, then

$$
\lim _{\epsilon \rightarrow 0^{+}} \frac{\epsilon\left\|q_{1}-q_{2}\right\|}{\alpha\left(\epsilon q_{1}, \epsilon q_{2}\right)}=0 .
$$

Therefore, if $q_{1}(x) \neq q_{2}(x)$ for some $x \in \mathbf{R}^{3}$ and the above limit is different from zero, then $S\left(q_{1}\right) \neq S\left(q_{2}\right)$.

Here,

$$
\left\|q_{1}-q_{2}\right\|=\sup _{u_{-}} \sup _{\substack{x \in \mathbb{R}^{3} \\ t \in \mathbb{R}}}\left|\int_{-\infty}^{\infty} R(x, t-r) *\left(q_{1}-q_{2}\right) u_{-}(x, r) d r\right|
$$

where $u_{-}$denotes any incoming free solution of (1) (with $q \equiv 0$ ), $R$ the Riemann function of (1) with $q \equiv 0$, and $*$ denotes spatial convolution, $\alpha\left(q_{1}, q_{2}\right)$ is given by a constant times

$$
\begin{aligned}
& \left(\left\|q_{1}\right\|_{\infty}^{1 / 3}\left\|q_{1}\right\|_{1}^{1 / 6}+\left\|q_{1}\right\|_{1}^{1 / 2}\left\|q_{1}\right\|_{\infty}^{1 / 2}\right)\left(\left\|q_{1}\right\|_{\infty}^{1 / 3}\left\|q_{1}\right\|_{1}^{2 / 3}+\left\|q_{1}\right\|_{1}\right) \\
& +\left(\left\|q_{2}\right\|_{\infty}^{1 / 3}\left\|q_{2}\right\|_{1}^{1 / 6}+\left\|q_{2}\right\|_{1}^{1 / 2}\left\|q_{2}\right\|_{\infty}^{1 / 2}\right)\left(\left\|q_{2}\right\|_{\infty}^{1 / 3}\left\|q_{2}\right\|_{1}^{2 / 3}+\left\|q_{2}\right\|_{1}\right) .
\end{aligned}
$$

ACKNOWLEDGements. We would like to thank the Department of Mathematics of the Federal University of Pernambuco (Recife) for financial support. Part of this research was completed there during a summer visit in 1976.

\section{REFERENCES}

1. G. Perla Menzala, On inverse scattering for the Klein-Gordon equation with small potentials, Funkcial. Ekvac. (to appear).

2. - On the inverse problem for three-dimensional potential scattering, J. Differential Equations 20 (1976), no. 1, 233-247. MR 52 \#11372.

3. J. M. Chadam, The asymptotic behavior of the Klein-Gordon equation with external potential. II, Pacific J. Math. 31 (1969), 19-31. MR 41 \#9531.

4. W. A. Strauss, Nonlinear scattering theory, Scattering theory in mathematical physics, J. A. LaVita and J. P. Marchand, editors, Reidel, Dordrecht, Holland, 1974.

5. Ju. M. Berezanskii, The uniqueness theorem in the inverse problem of spectral analysis for the Schrodinger equation, Trudy Moskov. Mat Obsc. 7 (1958), 3-62; English transl., Amer. Math. Soc. Transl. (2) 35 (1964), 167-235. MR 21 \#1 88.

6. V. G. Romanov, Integral geometry and inverse problems for hyperbolic equations, Springer Tracts in Nat. Philos., Vol. 26, (1974). Springer-Verlag, Berlin and New York.

7. C. S. Morawetz and W. A. Strauss, Decay and scattering of solutions of a nonlinear relativistic wave equation, Comm. Pure Appl. Math. 25 (1972), 1-31. MR 46 \#2239.

INSTITUTO DE MATEMÁTICA, UFRJ, CAIXA POSTAL 1835 ZC-00 20.000 RJ, RIO DE JANEIRO, BRAZIL 